Bundesgesundheitsbl $2011 \cdot 54: 1-3$ DOI 10.1007/s00103-010-1196-7

Online publiziert: 29. Dezember 2010

(c) Springer-Verlag 2011
U. Koch · A. Mehnert · M. Härter

Institut und Poliklinik für Medizinische Psychologie, Zentrum für Psychosoziale

Medizin, Universitätsklinikum Hamburg-Eppendorf, Hamburg

\section{Chronische körperliche Erkrankungen und psychische Komorbidität}
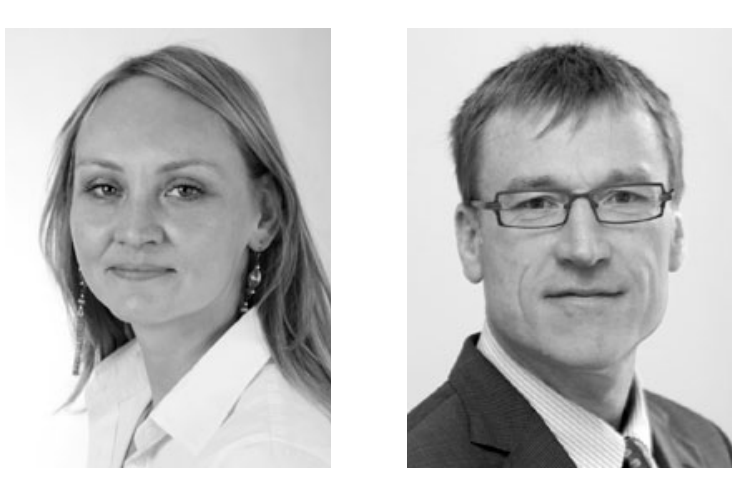

Liebe Leserin, lieber Leser,

als chronische körperliche Erkrankungen wird eine Vielzahl von somatischen Erkrankungen mit unterschiedlicher Ätiologie, Pathogenese, Symptomatik und Prognose bezeichnet. Dazu gehören Herz-Kreislauf-Erkrankungen und rheumatische Erkrankungen ebenso wie Krebserkrankungen und Erkrankungen, die eine Organ-oder Stammzelltransplantation zur Folge haben. Den chronischen Krankheitsbildern ist gemeinsam, dass sie langfristig bestehen und häufig progredient beziehungsweise mit phasenhaften Verschlimmerungen verlaufen. In der Regel wird bei chronischen Erkrankungen von einer multifaktoriellen Ätiologie ausgegangen. Dabei spielen sowohl eine besondere Disposition als auch häufig verhaltensbezogene Faktoren einschließlich Lebensstil und Gesundheitsverhalten einer Person eine zentrale Rolle.

Die Belastungsfaktoren, mit denen Menschen mit chronischen Erkrankungen konfrontiert sind, lösen unterschiedliche Reaktionen aus und werden je nach Art der Schädigung, nach Schweregrad, Intensität, Dauer, Lebensbedrohung etc. als unterschiedlich belastend erlebt. Aus diesen Belastungen können Einschränkungen der psychischen Befindlichkeit, der gesundheitsbezogenen Lebensqualität sowie der Aktivitäten des täglichen Lebens resultieren.

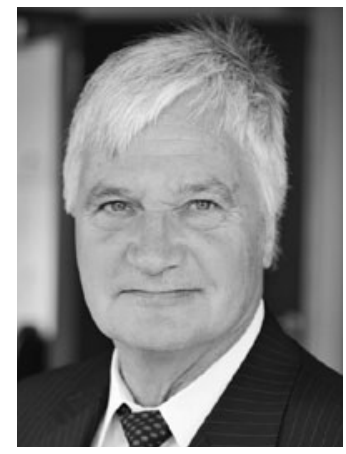

Die vielfältigen psychosozialen Folgen körperlicher chronischer Erkrankungen und deren Bewältigung waren in den letzten Jahrzehnten prioritärer Gegenstand medizinpsychologischer Forschung. Zu den häufigsten emotionalen Folgen der Erkrankungen gehören ein gestörtes emotionales Gleichgewicht, Ängste vor dem Wiederauftreten oder Fortschreiten der Erkrankung, Gefühle von Autonomie- und Kontrollverlust sowie von Hilf-und Hoffnungslosigkeit, Depressionen, Probleme durch ein verändertes Körperschema und Selbstkonzept sowie Selbstwert- und Identitätsprobleme. Die in diesem Kontext vorgelegten epidemiologischen Studien belegen, dass die Prävalenz psychischer Auffälligkeiten in Populationen körperlich Kranker sehr hoch ist. Einzelne Studien berichten einen Anteil von bis zu 50\% der Patienten, die behandlungsbedürftige psychische Beeinträchtigungen aufweisen, für die der Begriff komorbide psychische Störungen eingeführt wur- de. Gemeint ist damit, dass bei einem Patienten gleichzeitig eine somatische Erkrankung und psychische Störungen vorliegen. Das Spektrum solcher komorbider Störungen ist zum Teil diagnosenübergreifend, zum Teil von der Art der Erkrankung und den spezifischen medizinischen Therapien abhängig. Als gesichert gilt, dass chronisch körperlich Kranke mit einer psychischen Komorbidität eine schlechtere Lebensqualität, einen ungünstigeren Krankheitsverlauf aufweisen und vermutlich auch höhere Kosten im Gesundheitssystem verursachen.

Das hier vorgelegte Schwerpunktheft stellt die Thematik „Psychische Komorbidität bei chronischen körperlichen Erkrankungen" in den Mittelpunkt und bearbeitet das Thema in 16 Beiträgen aus unterschiedlichen Perspektiven.

Die beiden Eingangsbeiträge zielen darauf, mit definitorischen Klärungen die Grundlage zum Verständnis der nachfolgenden Beiträge zu schaffen. 
H. Raspe befasst sich in diesem Kontext mit dem Begriff „chronische Erkrankung". Er verweist auf die vielfältigen Erscheinungsformen chronischer Krankheiten und sieht als kleinsten gemeinsamen Nenner für alle Formen des chronischen Krankseins, dass die mit ihnen verbundenen Lasten die Betroffenen bis an ihr Lebensende begleiten werden. H. Baumeister, S. Kury und J. Bengel setzen sich danach in ihrem Beitrag mit der Definition und dem Verständnis von somatopsychischer Komorbidität auseinander. Die Autoren legen dar, wie sich das Konstrukt "Komorbidität“ gegenüber den verwandten Konstrukten „Multimorbidität“, „Krankheitsbelastung“ und "Patientenkomplexität“ abgrenzen lässt.

Die nachfolgenden vier Artikel befassen sich diagnosegruppenübergreifend mit Fragen der Epidemiologie, der Diagnostik und der Interventionsmöglichkeiten komorbider psychischer Störungen bei körperlichen Erkrankungen. In diesem Kontext geben zunächst M. Beutel und H. Schulz einen Überblick über die in vorliegenden epidemiologischen Studien berichteten Prävalenzraten für die psychische Komorbidität und beschreiben den möglichen Einfluss psychischer Erkrankungen auf Entstehung und Verlauf chronischer Erkrankungen am Beispiel der koronaren Herzerkrankungen. Der Beitrag von K. Reuter und M. Härter hebt die Notwendigkeit eines frühzeitigen Erkennens psychischer Belastungen bei chronischen Erkrankungen in der medizinischen Versorgung hervor. Die Autoren definieren die Voraussetzungen für eine valide Diagnostik solcher Störungen und stellen spezielle psychodiagnostische Instrumente sowohl für die dimensionale (Screeninginstrumente) als auch für die kategoriale Diagnostik (klinische Interviews) vor.

Im ersten der beiden auf Behandlung zielenden Beiträge setzen sich U. Koch, A. Mehnert und B. Strauß mit den psychologischen Interventionsmöglichkeiten bei chronisch körperlich kranken Patienten mit psychischer Komorbidität auseinander. Die Interventionsangebote werden nach Zielpopulationen, Versorgungsmodellen, Indikationen, Behandlungsmodellen und Versorgungsberei- chen differenziert und reichen von eher unspezifischen Ansätzen auf Basis allgemeiner psychosozialer Unterstützung über Gesundheitsförderung und Patientenschulung bis hin zu problemspezifischen Interventionen. G. Laux zeigt in seinem Beitrag die Möglichkeiten, aber auch die Limitationen psychopharmakologischer Interventionen bei psychischer Komorbidität auf. Im Artikel werden wichtige Hinweise aufgeeignete psychopharmakologische Behandlungsstrategien bei verschiedenen psychischen Beeinträchtigungen bei einzelnen körperlichen Erkrankungen gegeben und auf die Möglichkeiten kombinierter Behandlung von Psychopharmakotherapie und Psychotherapie verwiesen.

Die nachfolgenden fünf Beiträge setzen sich mit der psychischen Komorbidität bei einzelnen chronischen körperlichen Erkrankungen auseinander. Zunächst analysieren J. Weis und A. Boehncke das Problem der psychischen Komorbidität im Verlauf von Krebserkrankungen. Obwohl diese Fragestellung in der internationalen psychoonkologischen Forschung in den letzten Jahren intensiv beforscht wurde, schwanken die Prävalenzraten zwischen den einzelnen Untersuchungen erheblich. Die Autoren verweisen darauf, dass zu einer modernen onkologischen Versorgungskonzeption heute die Abklärung von beim Patienten bestehenden psychosozialen Belastungen und psychischer Komorbidität zu den zu fordernden Standards gehört. Anschließend befassen sich H. Baumeister und M. Härter mit der Epidemiologie, Ätiologie und Behandlung komorbider psychischer Störungen bei Patienten mit muskuloskelettalen Erkrankungen. Sie zeigen, dass bei Patienten mit muskuloskelettalen Erkrankungen ein deutlich erhöhtes Risiko für das Vorliegen einer komorbiden psychischen Störung im Vergleich zu Personen ohne diese Erkrankungen besteht. Für degenerative sowie entzündliche Gelenkerkrankungen und depressive Störungen wird beispielhaft ein mögliches Kausalmodell dargestellt.

K.H. Ladwig, R.T. Emeny, S. Häfner, und M.E. Lacruz setzen sich mit der Bedeutung der Depression als Risikofaktor bei der Entstehung und Progression der koronaren Herzerkrankung auseinander. Im Mittelpunkt steht dabei die Diskussion dreier unterschiedlicher zentraler psychobiologischer Pathomechanismen, nämlich die Annahme einer Hyperregulation des autonomen Nervensystems, die Annahme einer überschießenden Stressantwort des endokrinen Systems und die Annahme einer dauerhaften subklinischen Erhöhung von Inflammationsparametern.

M. Erhart, A. Weimann, M. Bullinger, M. Schulte-Markwort und U. Ravens-Sieberer untersuchen in ihrem Beitrag emotionale und Verhaltensprobleme und den psychosozialen Versorgungsbedarf bei Kindern und Jugendlichen mit chronisch somatischen Erkrankungen. Empirisch stützen sie sich dabei auf Daten des bundesweiten repräsentativen Kinder- und Jugendgesundheitssurveys (KiGGS). Die Autorinnen demonstrieren eindrucksvoll die Bedeutung sozioökonomischer, struktureller und funktionaler Aspekte der Familie für die Entwicklung und Prävention psychischer Probleme bei chronisch körperlich erkrankten Kindern und Jugendlichen.

A. Mehnert, J. Volkert, O. Wlodarczyk und S. Andreas fokussieren die psychische Komorbidität bei Menschen mit chronischen Erkrankungen im höheren Lebensalter und stellen dies insbesondere am Beispiel von Menschen mit einer Krebserkrankung dar. Gegenstand sind die Prävalenzraten für psychische Störungen im höheren Lebensalter im Allgemeinen und bei Patienten mit Krebserkrankungen, die spezifischen diagnostischen Möglichkeiten, die Risikofaktoren für die Entwicklung einer psychischen Erkrankung und deren Behandlungsmöglichkeiten in der Versorgung von älteren chronisch kranken Menschen.

Der sich anschließende Themenblock befasst sich mit der Bedeutung der psychischen Komorbidität in verschiedenen Versorgungsbereichen.

M. Rose, I. Wahl, J. Crusius und B. Löwe betrachten das Problem aus der Perspektive der stationären Akutversorgung. Sie zeigen auf, dass ein großer Anteil der körperlich Kranken, die in Akutkrankenhäusern behandelt wer- 
den, gleichzeitig an depressiven Erkrankungen, Angststörungen, somatoformen Störungen und Suchterkrankungen leidet, die aber im Krankenhaus häufig unerkannt oder unbehandelt bleiben. Die Autoren diskutieren die Voraussetzungen für eine verbesserte Versorgung von Akutpatienten.

M. Morfeld und A. Friedrichs setzen sich mit der Diagnostik und therapeutischen Berücksichtigung der psychischen Komorbidität im Rahmen der medizinischen Rehabilitation auseinander und illustrieren dies am Beispiel der muskuloskelettalen Erkrankungen. Die Autoren zeigen die besonderen Möglichkeiten des rehabilitativen Settings für den Einsatz von Screeningverfahren für die Erfassung psychischer Komorbidität auf und diskutieren potenzielle Barrieren aufseiten der Behandler, aber auch aufseiten der Patienten für die Anwendung von psychologischen Interventionen.

L. Pieper, J. Dirmaier, J. Klotsche, C. Thurau, D. Pittrow, H. Lehnert, W. März, U. Koch und U. Wittchen untersuchen auf der Basis von Daten einer Longitudinaluntersuchung, in die eine große Zahl von Hausarztpatienten einschlossen ist, Zusammenhänge zwischen depressiven Symptomen und Typ2-Diabetes sowie deren Auswirkungen auf die Mortalität. Die Untersuchung unterstreicht den Stellenwert depressiver Reaktionen im Hinblick auf den Verlauf der Erkrankung.

T. Fydrich und D. Ülsmann beschreiben aus der Perspektive der ambulanten Psychotherapie die Anforderungen an Psychotherapeuten, um Patienten mit komorbiden Störungen erfolgreich in diesem Handlungsfeld versorgen zu können. Sie verweisen darauf, dass über die bestehende Expertise für diese Störungen hinaus für die Psychotherapeuten die interdisziplinäre Kommunikation mit behandelnden Ärzten sowie fachübergreifende Grundkenntnisse von besonderer Bedeutung sind.

Der Schlussbeitrag von T. Lehnert, A. Konnopka, S. Riedel-Heller und H.-H. König behandelt gesundheitsökonomische Aspekte psychischer Komorbidität bei somatischen Krankheiten. Am Beispiel der komorbiden Depression bei
Diabetes mellitus zeigen sie, dass psychische Komorbidität mit erhöhten Behandlungskosten verbunden ist, die zumindest teilweise auf einer erhöhten Leistungsinanspruchnahme im Bereich der somatischen Versorgung beruhen.

Die Herausgeber des Themenheftes wünschen den Lesern und Leserinnen eine spannende Auseinandersetzung mit dem gewählten Thema.

Ihre
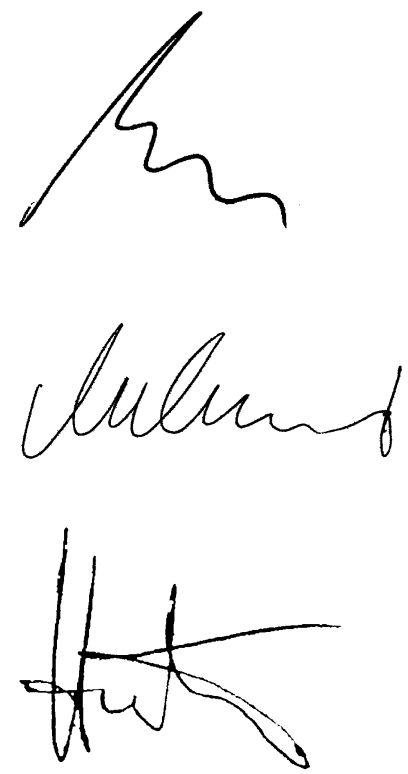

Uwe Koch, Anja Mehnert und Martin Härter

\section{Korrespondenzadresse}

\section{Prof. Dr. Dr. U. Koch}

Institut und Poliklinik für Medizinische Psychologie, Zentrum für Psychosoziale Medizin, Universitätsklinikum HamburgEppendorf Martinistraße 52, 20246 Hamburg koch@uke.de 